\title{
Comparative study in infertile couples with and without Chlamydia trachomatis genital infection
}

\author{
Yuanchang Zhu' ${ }^{1,2}$, Biao Yin ${ }^{3}$, Tonghua $\mathrm{Wu}^{4}$, Lijun Ye ${ }^{4}$, Chunmei Chen ${ }^{4}$, Yong Zeng ${ }^{4}$ and Yaou Zhang ${ }^{2 *}$
}

\begin{abstract}
Background: Chlamydia, caused by the bacterium Chlamydia trachomatis(C. trachomatis), is the most common sexually transmitted disease. The incidence is not clear due to the asymptomatic nature of early stage of infections. The incidence of Chlamydia has not been fully investigated in the Chinese Han population. Since chronic infection with can C. trachomatis can lead to infertility in males and females, it is important to determine the impact of infection on clinical outcomes. The aim of this study is to explore the epidemiology of $C$. trachomatis in subfertile couples and to determine whether infections will adversely affect clinical outcomes after assisted reproduction technique (ART) treatment.

Methods: Subfertile patients $(n=30760)$ were screened in the research for $C$. trachomatis in our center from January 2010 to December 2014. C. trachomatis-specific DNA was detected by Taq-man PCR from semen or swabs from the urethral, endocervix or vaginal. The control group consisted of 1140 subfertile patients without C. trachomatis infection. The prevalence and characteristics of $C$. trachomatis were identified for subfertile couples and clinical outcomes were collected and analyzed. A retrospective study was performed.
\end{abstract}

Results: Nine hundred and seventy patients were diagnosed with C. trachomatis infection, and the overall prevalence was $3.15 \%$ in the most recent five years, with a yearly increasing. The incidence was a higher in the second half of the year (3.40\%) compared to the first half (2.69\%). The age group with the highest-risk of infection with C. trachomatis was between 26 to 35 years old, and in about one third of the couples, both partners were infected. The basic parameters and clinical outcomes were not statistically significant between different the groups $(P>0.05)$, even though some minor data were different $(P<0.05)$.

Conclusions: $C$. trachomatis is a common infection in subfertile people and it is essential to test for this organism in ART couples' screening. This study identified no adverse on clinical outcomes after successful treatment of $C$. trachomatis infection, regardless of gender, age and number of C. trachomatis copies.

Keywords: Chlamydia trachomatis, Subfertile, Epidemiology, Assisted reproduction technique, Clinical outcome

\section{Plain English summary}

One of the most common bacterial sexually transmitted disease is Chlamydia, caused by Chlamydia trachomatis (C. trachomatis), the exact incidence of Chlamydia is unknown because the infection is often asymptomatic in the early stage. The incidence of Chlamydia it has not been fully investigated in the Chinese Han population.

\footnotetext{
* Correspondence: zhuyc14@mails.tsinghua.edu.cn

${ }^{2}$ Key Lab in Healthy Science and Technology, Division of Life Science,

Graduate School at Shenzhen, Tsinghua University, Shenzhen 518000, China

Full list of author information is available at the end of the article
}

Since chronic infection with C. trachomatis can induce infertility in males and females. The aim of the study is to explore the epidemiology of C. trachomatis in subfertile couples and to determine adverse effects on clinical outcomes after assisted reproduction technique (ART) treatments.

A total of 30760 subfertile patients were tested for $C$. trachomatis in our center from January 2010 to December 2014. C. trachomatis-specific DNA was detected by Taqman PCR. The prevalence and characteristics of C. trachomatis were identified for subfertile couples and clinical 
outcomes were collected and analyzed. A retrospective study was performed.

The overall prevalence of C. trachomatis was 3.15\% in a recent five year periods, with a yearly increase. The incidence was slightly higher in the second half of the year (3.40\%) compared to the first half of the year $(2.69 \%)$. The age range with the highest-risk infection of of $C$. trachomatis was between 26 and 35 years, and in about one third of the couples, both partners had the infection. The main parameters and clinical outcomes were not statistically significant between the groups $(P>0.05)$, even though some minor data were different $(P<0.05)$.

The results of this ananlysis confirm that of C. trachomatis is common infection in subfertile people and it is essential to test for the presence of this microbe as part of ART couples's screening. This study found no adverse effects on clinical outcomes after the infection was cured.

\section{Background}

Chlamydia trachomatis (C. trachomatis), an intracellular bacterium, is the cause of the most frequently sexually transmitted disease (STD), resulting in about 100 million new cases annually worldwide [1]. Previous studies have reported that the prevalence of $C$. trachomatis ranged from $1.7 \%$ to $17 \%$ among European women [2] and from 1\%-40\% among asymptomatic men with a longstanding history of couple infertility [3], depending on the method of diagnosis, social background, area of residence, occupation, gender, and among others. Approximately $50 \%$ males and $80 \%$ females with Chlamydia show no symptoms in the early stage of infection, consequently, many of these individuals remain undiagnosed and are often poorly or inadequately treated [4]. Therefore, the true prevalence of this infection is not clear, and may remain unknown for several years or even longer. Standard microbiologic tests were commonly used to diagnosis C. trachomatis infections in the past, but studies have proved that this method may fail to reveal the pathogen, owing to high-proportion false negative results [5]. Thus, the DNA amplification method is increasingly universal. This method has a high sensitivity (99\%) and specificity (100\%), and has been accepted as the gold standard for the identification of $C$. trachomatis infections. [6, 7].

Since C. trachomatis is often asymptomatic and treatment is often delayed, the infection is often transmitted to sexual partner(s) [8]. It can cause fallopian tube salpingitis, cervicitis, and pelvic inflammatory disease (PID), which in turn can cause infertility and ectopic pregnancy in women with chronic infections [9].

Research has shown that microbiologic tests done on semen revealed a high proportion (43\%) of $C$. trachomatis -specific DNA in semen from men with azoospermia [10], suggesting that hronic progression of $C$. trachomatis in the male genital tract may lead to urethritis, epididymitis, and even obstructive azoospermia [11]. Some subfertile couples are seeking ART treatment due to STD infection.

Some studies report that the subfertile men with genitourinary infection caused by $C$. trachomatis had increased sperm DNA fragmentation [12], leading to sperm apoptosis [13] and low to sperm quality [10]. In animal experiments, C. trachomatis infection has been shown to inhibit oocyte transport in the mouse oviduct [14]. These studies suggest that C. trachomatis infection has the potential to negatively affect gamete formation and development. Despite the advances in knowledge about C. trachomatis infection, the impact of prior infection C. trachomatis on clinical outcomes after successful treatment is unknown. Therefore, the present study was carried out to evaluate C. trachomatis infection in subfertile couples I south China that were diagnosed by Taq-man PCR, and to deteremine whether the infection has any adverse effects on the clinical outcomes after treatment is completed.

\section{Methods \\ Study population}

The study patients were recruited from couples of reproductive age complaining of primary or secondary infertility that came to our reproductive center for ART treatment, from January 2010 to December 2014. For the study, a total of 30760 patients were screened for epidemiology statistical analysis. After fulfilling the eligibility criteria, 970 patients were diagnosed with $C$. trachomatis infection and 761 of these were included for clinical outcomes analysis. Subfertile $(n=1140)$ patients without $C$. trachomatis infection were recruited as the control group at corresponding period. This study was approved by the Research Ethics Committee of Shenzhen Zhongshan Urology Hospital, and informed consent was obtained from all couples prior to the study.

\section{Sample collection and C. trachomatis detection}

A total of 30760 testing samples (including endocervical swab, semen and urethral swab) were obtained. Samples were collected into sterile containers and transported to the laboratory at room temperature within 2 hours. The DNA was extracted within 24 hours, according to instructions provided in commercial kit (Sansure Biotech, China). The endocervical and urethral swabs were placed in $1 \mathrm{ml}$ normal saline; semen samples $(50 \mu \mathrm{l})$ were added to $950 \mu \mathrm{l}$ normal saline, and diluted $(20 \times)$; the samples were sufficiently mixed by vortex for $15 \mathrm{~s}$. Aliquots $(0.5 \mathrm{ml})$ were centrifuged 


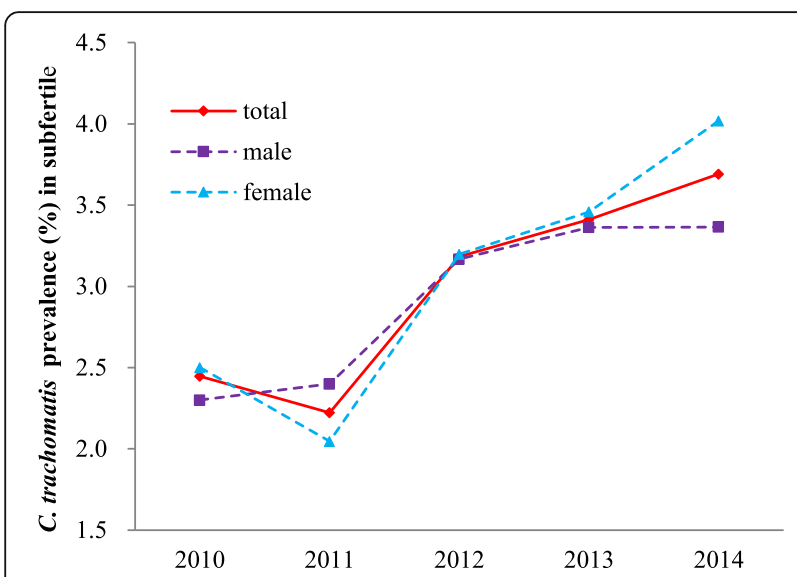

Fig. 1 The incidence of $C$. trachomatis in subfertile individuals from 2010 to 2014

at $12000 \mathrm{rpm}$ for $10 \mathrm{~min}$, and the supernatants discarded. The pellets underwent lysis, mixed thoroughly and waited for $10 \mathrm{~min}$ in room temperature, and were stored at $-20^{\circ} \mathrm{Cuntil}$ PCR analysis was performed. We performed four different concentration reference standards (from $10^{4}$ to $10^{7}$ ) to generate draw the standard curve, and then calculated the bacterial copy number for positive infection samples using absolute quantification real-time PCR methods. The kit for in vitro clinical diagnosis relies on absolute quantification of C. trachomatis burden (using FAM-labeled Taq-man primer-probe set), as assessed by the inclusion of known $C$. trachomatis standards. Furthermore, this system includes a Vic-labeled internal standard primer-probe set to test for the presence of PCR inhibitors. The PCR amplification was assessed using the ABI PRISM 7500 Sequence Detection System (Applied Biosystems, Foster City, CA). The assay set up internal control, positive control and negative control. The sample was deemed as positive if the number was higher than $200 / \mathrm{ml}$ copies.

\section{Ovarian stimulation protocol}

All female patients received the luteal-phase gonadotrophinreleasing hormone agonist protocol. Since the pituitary was adequately suppressed (triptorelin acetate injection; Tiantaishan Pharmaceuticals, China), ovarian stimulation was induced with human menopausal gonadotrophin (Menotropins for Injection; Livzon, China) and recombinant FSH (Gonal-F; Merck Serono, Switzerland). Human chorionic gonadotrophin (hCG; Chorionic Gonadotrophin for Injection; Livzon, China) was injected when two or more dominant follicles reached $18 \mathrm{~mm}$ in diameter. Oocytes were collected $36 \mathrm{~h}$ after hCG administration by vaginal ultrasound guided follicular aspiration.

\section{Embryo culture, transfer and pregnancy evaluation}

Fertilization was taken 16-18 h after IVF (day 1; oocytes retrieved day defined as day 0 ) by the presence of pronuclei. Zygotes were cultured in Quinn's Advantage Cleavage Medium (Quinn's 1026; SAGE, USA) containing $10 \%(\mathrm{v} / \mathrm{v})$ serum protein substitute (SAGE). On day 3 , embryos were scored using morphological criteria as previously described [15]. Grade 1 and 2 embryos were defined as high-grade embryos. Two or three cleavagestage embryos (at least one high-grade embryo) were selected for transfer and the remaining embryos were stored in liquid nitrogen. The clinical pregnancy was identified by ultrasound examination at 5 weeks after the embryo transferr. The fertilization rates, cleavage rates, clinical pregnancy rates, implantation rates, miscarriage rates, take baby home rates and other data were collected and analyzed.

\section{Statistical analysis}

Continuous data were presented as mean \pm standard deviation (SD) and percentage. Mann-Whitney U test, Pearson's chi-squared or Fisher's exact tests were used to determine the difference. The statistical analyses

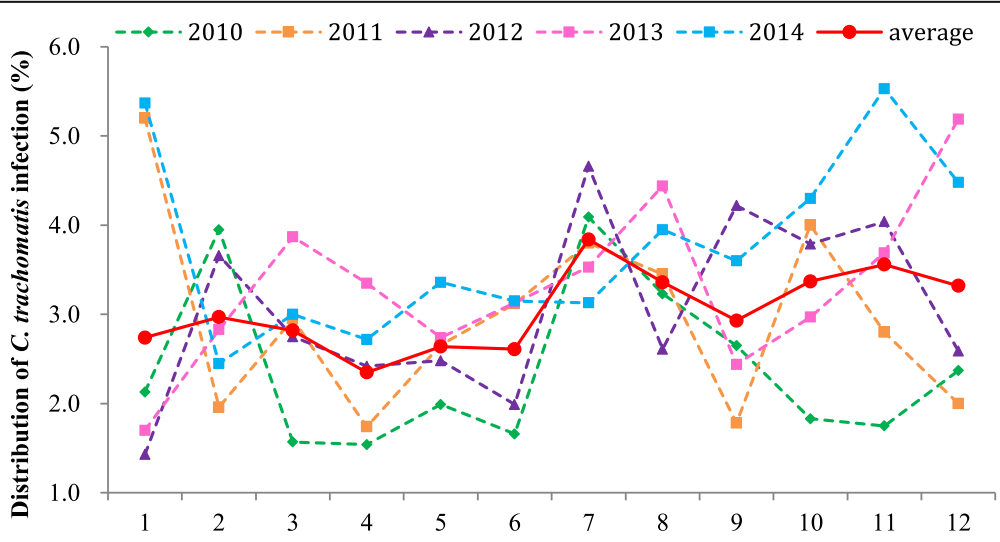

Fig. 2 The prevalence and distribution of $C$. trachomatis infection in recent five years during different months 


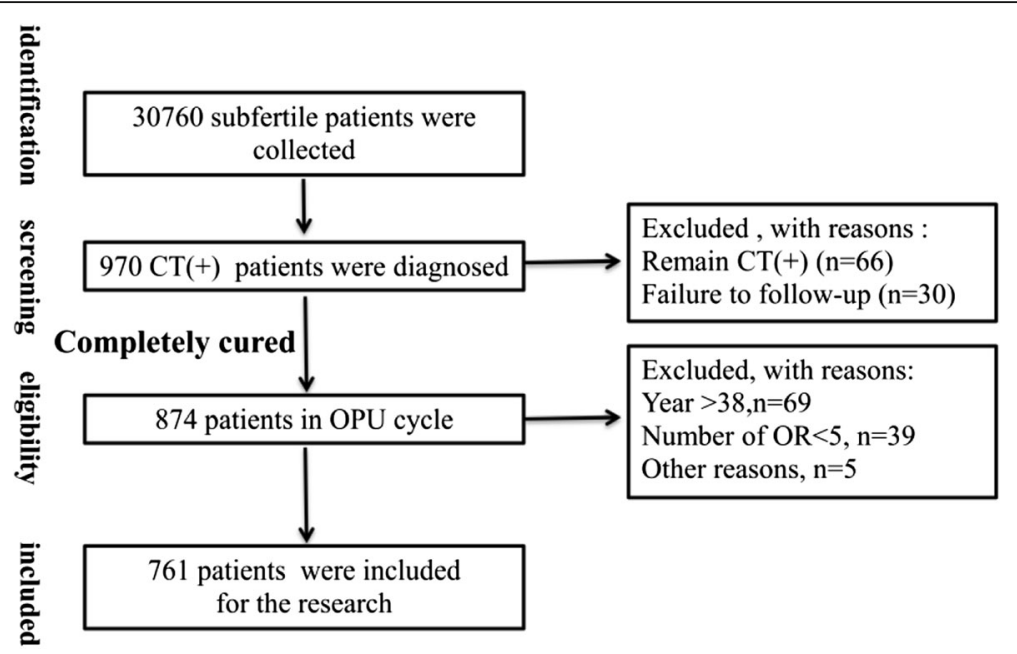

Fig. 3 Schematic of the recruitment in the study. Abbreviation: C. trachomatis, Chlamydia trachomatis; OR, oocyte retrieve; OPU, oocyte pick-up

were performed by SPSS 17.0 software (SPSS Inc, Chicago, IL, USA). $P<0.05$ was considered statistically significant.

\section{Results}

Prevalence of $C$. trachomatis for subfertile patients

A total of 30760 samples were collected for the study over a period of five years and 970 patients identified as positive by PCR diagnostics, giving a prevalence rate of $3.15 \%$ (Fig. 1). The prevalence rate was $2.45 \%$ in 2010 and was $3.69 \%$ in 2014, showing an increasing trend year by year. The incidence was similar between the two genders $(P>0.05)$.

\section{Prevalence of $C$. trachomatis during different times of} the year

As shown in Fig. 2, the incidence of C. trachomatis was correlated with different seasons, in the past five years, the incidence was $3.40 \%$ in the second half year, it was slightly higher than the first half year $(2.69 \%)$, and the difference was statistically significant $(P<0.05)$.

Trails eligible for inclusion in clinical outcomes analysis After identification, and eligibility screening, 761 patients (including single and dual infection of subfertile couples) were included in the study for clinical outcome

Table 1 Parameters and clinical outcomes of patients with and without C. trachomatis infection

\begin{tabular}{|c|c|c|c|c|}
\hline Variables & C. trachomatis $(+)$ & C. trachomatis (-) & $P$ value & OR $(95 \% \mathrm{Cl})$ \\
\hline No. of couples & 761 & 1140 & / & \\
\hline Male age (mean[IQR]) & $33.40[29 .-35]$ & $33.79[27-35]$ & 0.874 & \\
\hline Female age (mean[IQR]) & $30.87[24-32]$ & $30.71[25-32]$ & 0.342 & \\
\hline Duration of infertility[IQR] & $4.00[2-5]$ & $3.76[2-5]$ & 0.223 & \\
\hline Oocytes retrieved [IQR] & $14.73[10-19]$ & $14.68[9-19]$ & 0.529 & \\
\hline MII rate & $88.80(9957 / 11213)$ & $88.82(14873 / 16746)$ & 0.966 & $0.998(0.925-1.077)$ \\
\hline Fertilized rate & $76.55(8584 / 11213)$ & 75.98 (12724/16746) & 0.271 & $1.032(0.976-1.021)$ \\
\hline Cleaved rate & $96.28(8265 / 8584)$ & $96.03(12219 / 12724)$ & 0.348 & $1.071(0.928-1.235)$ \\
\hline Transfer cycles & 643 & 938 & / & \\
\hline Transferred embryos[IQR] & 2. $32[2,3]$ & $2.25[2-5]$ & 0.036 & \\
\hline Embryo implantation rate & $32.84(490 / 1492)$ & $36.56(770 / 2106)$ & 0.021 & $0.848(0.738-0.976)$ \\
\hline Clinical pregnancy rate & $52.88(340 / 643)$ & $54.69(513 / 938)$ & 0.477 & $0.930(0.760-1.137)$ \\
\hline Miscarriage rate & $13.24(45 / 340)$ & $16.18(83 / 513)$ & 0.238 & $0.790(0.534-1.169)$ \\
\hline Multiple pregnancy rate & $43.82(149 / 340)$ & $46.78(240 / 513)$ & 0.395 & $0.887(0.674-1.169)$ \\
\hline Take baby home rate & $45.88(295 / 643)$ & 45.95 (431/938) & 0.978 & $0.997(0.815-1.220)$ \\
\hline
\end{tabular}

Note: + = previously-positive C. trachomatis; IQR interquartile range 
Table 2 Parameters and clinical outcomes in patients cured of C. trachomatis by gender

\begin{tabular}{|c|c|c|c|c|}
\hline Variables & $\begin{array}{l}\text { Male } \\
\text { C. trachomatis (+) }\end{array}$ & $\begin{array}{l}\text { Female } \\
\text { C. trachomatis (+) }\end{array}$ & $P$ & OR $(95 \% \mathrm{Cl})$ \\
\hline No. of couples & 374 & 387 & / & \\
\hline Male age (mean[IQR]) & $33.26[30-36]$ & 33.53 [29-36] & 0.670 & \\
\hline Female age (mean[IQR]) & 30.77 [28-33] & $30.97[28-34]$ & 0.916 & \\
\hline Duration of infertility[IQR] & $4.00[2-5]$ & $4.01[2-5]$ & 0.180 & \\
\hline Oocytes retrieved [IQR] & $14.50[10-18]$ & $15.00[10-39]$ & 0.420 & \\
\hline MIl rate & $87.72(4756 / 5423)$ & $89.60(5201 / 5805)$ & 0.002 & $0.828(0.737-0.931)$ \\
\hline Fertilized rate & $76.29(4137 / 5423)$ & $76.61(4447 / 5805)$ & 0.689 & $0.982(0.900-1.072)$ \\
\hline Cleaved rate & $96.74(4002 / 4137)$ & $95.86(4263 / 4447)$ & 0.032 & $1.280(1.020-1.604)$ \\
\hline Transfer cycles & 307 & 336 & / & \\
\hline Transferred embryos [IQR] & 2. $29[2,3]$ & $2.35[2,3]$ & 0.105 & \\
\hline Embryo implantation rate & $34.14(240 / 703)$ & $31.69(250 / 789)$ & 0.314 & $1.118(0.900-1.387)$ \\
\hline Clinical pregnancy rate & $54.72(168 / 307)$ & $51.19(172 / 336)$ & 0.370 & $1.152(0.845-1.572)$ \\
\hline Miscarriage rate & $13.10(22 / 168)$ & $13.37(23 / 172)$ & 0.940 & $0.976(0.521-1.828)$ \\
\hline Multiple pregnancy rate & $42.86(72 / 168)$ & $44.778(77 / 172)$ & 0.723 & $0.925(0.603-1.420)$ \\
\hline Take baby home rate & $49.51(152 / 307)$ & $44.64(150 / 336)$ & 0.236 & $1.216(0.892-1.659)$ \\
\hline
\end{tabular}

Note: $+=$ previously-positive C. trachomatis

statistical analysis, (Fig. 3). Meanwhile, 1140 subfertile couples without $C$. trachomatis infection were enrolled for the control group.

\section{Clinical outcomes in C. trachomatis infection}

The basic parameters (Table 1), such as the age of couples and duration of infertility, were similar between the two groups $(P>0.05)$. The number of transferred embryos was $2.32 \pm 0.59$ in the C. trachomatis group and $2.25 \pm 0.64$ in control group, and the difference was statistically significant $(P<0.05)$. The implantation rate in $C$. trachomatis group was $32.84 \%$, which was significantly lower than in control group $(P<0.05)$, which had a rate of $36.56 \%$. The fertilization rate, cleaved rate, multiple pregnancy rate, clinical pregnancy rate, miscarriage rate and take-home baby rate were comparable between the two groups $(P>0.05)$.

\section{Clinical outcomes in different genders}

A total of 761 C. trachomatis infection patients were recruited in the study for clinical outcome analysis. These patients underwent successful treatment for the infections. They were further divided into two subgroups: male (374 patients) and female subgroup (387 patients) respectively. In the male subgroup, the MII rate was significantly lower but the cleaved rate was significantly higher, both differences were reached statistically significance $(P<0.05)$, when compared to the female subgroup. All other clinical parameters were not significantly different between genders $(P>0.05)$ (As shown in Table 2$)$.

\section{Distribution and clinical outcomes stratified by age}

Patients were divided into four subgroups according to age: $\leq 25$ years; $26-30$ years; $31-35$ years and $>35$ years. As shown in Fig. 4, the C. trachomatis constituent ratio different between the age groups. The age group having the highest prevalence rate was 31-35(38.12\%), followed by $26-30(30.22 \%),>35(26.10 \%)$ and $\leq 25$ years $(5.56 \%)$. The basic parameters and clinical outcomes were comparable $(P>0.05)$ in different age brackets, even though the miscarriage rate was higher and the take-home baby rate were slight lower in advanced age group $(P<0.05)$ (Table 3$)$.

\section{Distribution and clinical outcomes versus $C$. trachomatis copy number}

The $C$. trachomatis copy numbers were mainly in the range of $10^{3} \sim 10^{6}$ copies (Fig. 5), accounting for

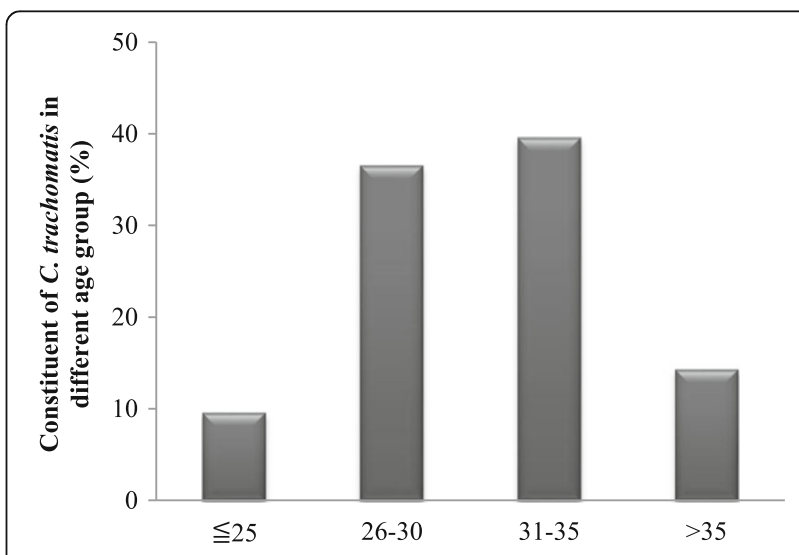

Fig. 4 The distribution of C. trachomatis in different age groups 
Table 3 Parameters and Clinical outcomes of C. trachomatis in different age groups

\begin{tabular}{|c|c|c|c|c|}
\hline Variables & $\leq 25$ & $26-30$ & $31-35$ & $35-38$ \\
\hline No. of couples & 73 & 278 & 301 & 109 \\
\hline Duration of infertility [IQR] & 2.89 [2-5] & $3.48[2-6]$ & $4.21[2-6]$ & $5.50[3-8]$ \\
\hline Oocytes retrieved [IQR] & 16.71 [10-20] & $15.8[10-18]$ & $13.94[9-19]$ & $13.03[9-18]$ \\
\hline MIl rate & $90.9(1110 / 1220)$ & $88.0(3855 / 4378)$ & $89.8(3769 / 4195)$ & $86.1(1223 / 1420)$ \\
\hline Fertilized rate & $77.0(940 / 1220)$ & $76.9(3371 / 4378)$ & $76.8(3223 / 4195)$ & $73.9(1050 / 1420)$ \\
\hline Cleaved rate & $95.9(902 / 940)$ & $96.4(3250 / 3371)$ & $96.2(3103 / 3223)$ & $96.1(1010 / 1050)$ \\
\hline Transfer cycles & 67 & 223 & 253 & 101 \\
\hline Transferred embryos [IQR] & $2.30[2,3]$ & $2.20[2,3]$ & $2.36[2,3]$ & $2.50[2,3]$ \\
\hline Embryo implantation rate & $34.4(53 / 154)$ & $34.0(167 / 490)$ & $31.2(186 / 595)$ & $33.2(84 / 253)$ \\
\hline Clinical pregnancy rate & $52.2(35 / 67)$ & $52.9(118 / 223)$ & $52.1(132 / 253)$ & $55.4(56 / 101)$ \\
\hline Miscarriage rate & $14.2(5 / 35)$ & $7.6(9 / 118)$ & $12.1(16 / 132)$ & $28.5(16 / 56)$ \\
\hline Multiple pregnancy rate & $51.4(18 / 35)$ & $41.5(49 / 118)$ & $40.9(54 / 132)$ & $50.0(28 / 56)$ \\
\hline Take baby home rate & $44.7(30 / 67)$ & $48.8(109 / 223)$ & $45.8(116 / 253)$ & $39.6(40 / 101)$ \\
\hline
\end{tabular}

approximately $80 \%$ of cases. Patients received ART treatment only after thereapy and the successful cure of infection. The longer the duration of infertility, the more embryos were transferred $(P<0.05)$, the other clinical parameters were similar among the groups $(P>0.05)$ (Table 4$)$.

\section{Distribution and clinical outcomes of one-sided or two- sided infections}

For C. trachomatis positive couples, some were merely one-sided (one partner infected), while other couples had two sided infections (both partners infected). We found that on third of $C$. trachomatis cases were two-sided infections, and the remainder was one-sided. However, as long as the treatment was successful, all the clinical outcomes were not statistically different between the two groups $(P>0.05)$ (Table 5 and Fig. 6).

\section{Discussion}

Infertility is becoming an increasingly significant health problem in both developed and developing countries, undoubtedly, including China. The increase appears to coincide with the growing role played by sexually transmitted disease [16]. Here, we found that C. trachomatis infection has no adverse effect on clinical outcomes after C. trachomatis was thoroughly cured. This is one of the largest epidemiological studies of genital C. trachomatis in assisted reproductive technology patients thus far.

The prevalence of C. trachomatis in subfertile couples in this study was $3.15 \%$, and increased yearly from $2.45 \%$ in 2010 to $3.69 \%$ in 2014 . It is slightly lower than previous published reports, which showed varied prevalence rates of between 1\% and 93.5\% [17-19]. Discrepancies of the findings in these studies might be due to the fact that almost all of the sub-infertile patients in our study have a regular sexual partner, and were asymptomatic prior to the study. In other study, many

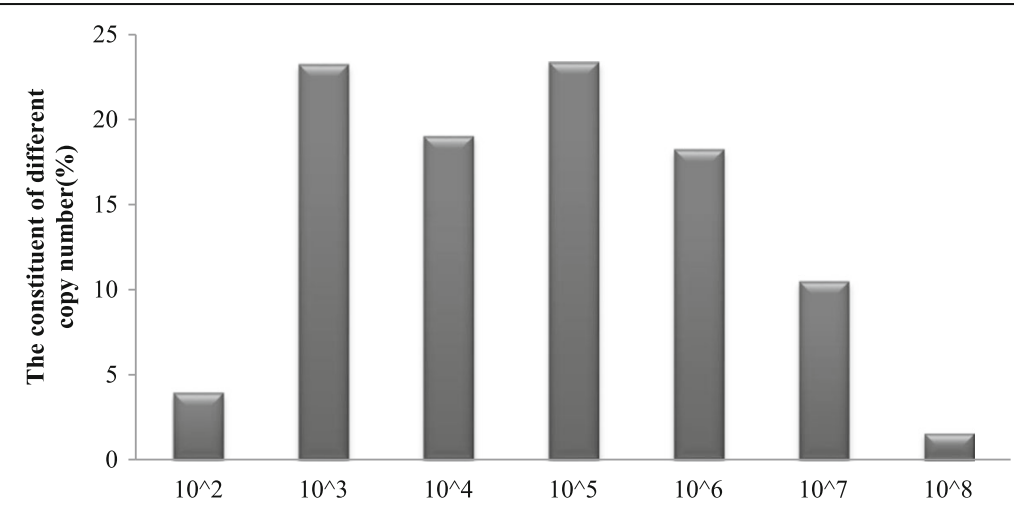

Fig. 5 C. trachomatis DNA copies $/ \mathrm{ml}$ in subfertile couples prior to treatment 
Table 4 Parameters and clinical outcomes for different C. trachomatis copy numbers

\begin{tabular}{llllllll}
\hline Variables & $10^{2}$ & $10^{3}$ & $10^{4}$ & $10^{5}$ & $10^{6}$ & $10^{7}$ & $10^{8}$ \\
\hline No. of couples & 30 & 177 & 145 & 178 & 139 & 80 & 12 \\
Male age & $34.50 \pm 5.32$ & $33.70 \pm 4.73$ & $33.59 \pm 4.29$ & $32.87 \pm 4.28$ & $33.59 \pm 5.02$ & $33.01 \pm 4.12$ & $31.83 \pm 4.65$ \\
Female age & $31.57 \pm 3.95$ & $30.92 \pm 4.17$ & $31.44 \pm 3.87$ & $30.22 \pm 4.11$ & $30.95 \pm 3.89$ & $30.88 \pm 3.58$ & $30.42 \pm 4.74$ \\
Duration of infertility & $3.07 \pm 2.34$ & $4.39 \pm 3.05$ & $4.04 \pm 2.76$ & $3.74 \pm 2.72$ & $4.12 \pm 3.20$ & $4.04 \pm 2.54$ & $2.50 \pm 1.17$ \\
Oocyte retrieve & $14.67 \pm 6.00$ & $15.28 \pm 6.48$ & $14.02 \pm 6.10$ & $15.05 \pm 6.57$ & $14.47 \pm 6.18$ & $14.71 \pm 6.36$ & $13.92 \pm 5.65$ \\
MIl rate & 90.0 & 88.2 & 89.6 & 88.2 & 90.2 & 88.0 & 80.8 \\
& $(396 / 440)$ & $(2387 / 2705)$ & $(1823 / 2033)$ & $(2365 / 2679)$ & $(1815 / 2012)$ & $(1036 / 1177)$ & $(135 / 167)$ \\
Fertilized rate & 76.1 & 76.0 & 78.1 & 75.6 & 78.9 & 73.2 & 76.0 \\
& $(335 / 440)$ & $(2056 / 2705)$ & $(1589 / 2033)$ & $(2026 / 2679)$ & $(1589 / 2012)$ & $(862 / 1177)$ & $(127 / 167)$ \\
Cleaved rate & 97.9 & 96.6 & 96.9 & 95.9 & 95.5 & 95.4 & 98.4 \\
& $(328 / 335)$ & $(1987 / 2056)$ & $(1540 / 1589)$ & $(1943 / 2026)$ & $(1519 / 1589)$ & $(823 / 862)$ & $(125 / 127)$ \\
Transfer cycles & 20 & 156 & 125 & 145 & 123 & 63 & 11 \\
Transferred embryos & $2.10 \pm 0.45$ & $2.64 \pm 0.51$ & $2.30 \pm 0.63$ & $2.22 \pm 0.60$ & $2.20 \pm 0.58$ & $2.29 \pm 0.61$ & $2.36 \pm 0.51$ \\
Embryo implantation rate & $33.3(14 / 42)$ & $32.0(128 / 400)$ & $31.5(91 / 288)$ & $32.6(105 / 322)$ & $33.7(91 / 270)$ & $31.9(46 / 144)$ & $57.6(15 / 26)$ \\
Clinical pregnancy rate & $45.0(9 / 20)$ & $55.1(86 / 156)$ & $54.4(68 / 125)$ & $51.0(74 / 145)$ & $52.0(64 / 123)$ & $47.6(30 / 63)$ & $81.8(9 / 11)$ \\
Miscarriage rate & $11.1(1 / 9)$ & $10.4(9 / 86)$ & $14.7(10 / 68)$ & $10.8(8 / 74)$ & $15.6(10 / 64)$ & $15.2(7 / 46)$ & $0.0(0 / 9)$ \\
Multiple rates & $55.5(5 / 9)$ & $48.8(42 / 86)$ & $33.8(23 / 68)$ & $41.8(31 / 74)$ & $42.1(27 / 64)$ & $34.7(16 / 46)$ & $55.5(5 / 9)$ \\
Take baby home rate & $40.0(8 / 20)$ & $49.3(77 / 156)$ & $46.4(58 / 125)$ & $45.5(66 / 145)$ & $43.9(54 / 123)$ & $36.5(23 / 63)$ & $81.8(9 / 11)$ \\
\hline
\end{tabular}

subjects had symptoms, such as warts, ulcers, ectopy, lesions, erythema and discharge; some had more than one sexual partner. Other different between our study and others include the criteria and sample size of patients, area of residence, occupation, gender, diagnostic methods, specimen type and clinical study design.

The positive rate of $C$. trachomatis was $2.92 \%$ in males and $3.04 \%$ in females, respectively, which is slightly higher than previous reports [20]. The incidence in different genders is close [21], in contrast to other indications of higher rates in females than in males [22]. In these literatures, the subjects are mainly women involved in prostitution; females carry an increased risk for infection based on their anatomy as well. In contrast, the majority of patients in this study have a regular lifetime partner, thus the risk of sexually transmitted infections is relatively lower.

In the C. trachomatis positive group, the number of transferred embryos was significantly higher, while the

Table 5 Baseline demographics and clinical outcomes for different C. trachomatis copy numbers

\begin{tabular}{|c|c|c|c|c|}
\hline Variables & $\begin{array}{l}\text { Side two } \\
\text { C. trachomatis (+) }\end{array}$ & $\begin{array}{l}\text { Side one } \\
\text { C. trachomatis (+) }\end{array}$ & $P$ value & OR $(95 \% \mathrm{Cl})$ \\
\hline No. of couples & 271 & 490 & / & \\
\hline Male age & $33.28[28-36]$ & $33.46[30-36]$ & 0.841 & \\
\hline Female age & $30.87[27-34]$ & $30.88[28-34]$ & 0.996 & \\
\hline duration & $4.04[2-5]$ & $3.98[2-5]$ & 0.828 & \\
\hline Oocyte retrieve & 13.69[9-17] & $15.31[11-19]$ & 0.000 & \\
\hline MIl rate & $88.79(3294 / 3710)$ & $88.83(6663 / 7501)$ & 0.948 & $0.996(0.879-1.128)$ \\
\hline Fertilized rate & $75.61(2805 / 3710)$ & $77.04(5779 / 7501)$ & 0.091 & $0.924(0.842-1.013)$ \\
\hline Cleaved rate & $96.74(2716 / 2805)$ & 96.026 (5549/5779) & 0.064 & $1.265(0.986-1.622)$ \\
\hline Transfer cycles & 235 & 408 & / & \\
\hline Transferred embryos & 2. $33[2,3]$ & $2.32[2,3]$ & 0.694 & \\
\hline Embryo implantation rate & $31.08(170 / 547)$ & $33.86(320 / 945)$ & 0.270 & $0.881(0.703-1.104)$ \\
\hline Clinical pregnancy rate & $51.49(121 / 235)$ & $53.92(220 / 408)$ & 0.552 & $0.907(0.658-1.251)$ \\
\hline Miscarriage rate & $14.88(18 / 121)$ & $12.27(27 / 220)$ & 0.497 & $1.249(0.657-2.375)$ \\
\hline Multiple rates & $40.50(49 / 121)$ & $45.00(99 / 220)$ & 0.422 & $0.832(0.530-1.304)$ \\
\hline Take baby home rate & $43.83(103 / 235)$ & $47.06(192 / 408)$ & 0.429 & $0.878(0.636-1.212)$ \\
\hline
\end{tabular}




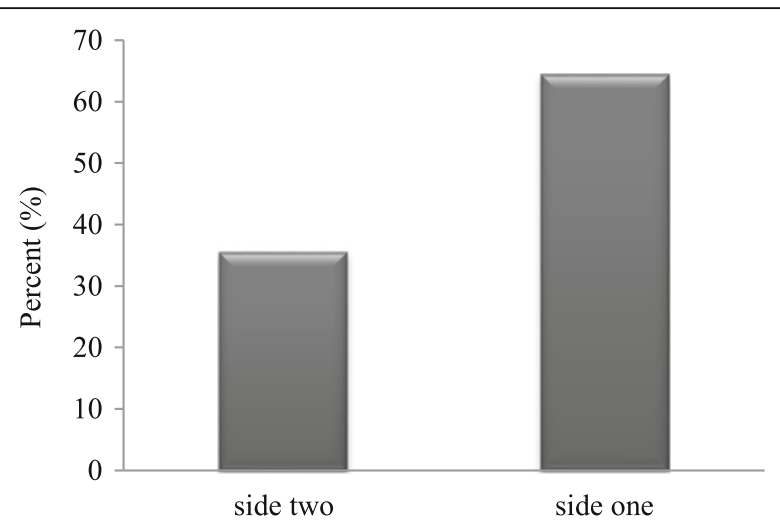

Fig. 6 The constituent ratios of subfertile couples with $C$. trachomatis infection

embryo implantation rate was lower than the control group. A possible explanation is that the female age was higher and the duration was longer, even though these differences were not statistically significant $(P>0.05)$.

Some researchers have found that the prevalence of $C$. trachomatis is the highest in younger people especially those under the age of 25 years [23]. The highest prevalence rate in our study was in women aged 25-35 years, in males and females. However, the average age of patients in our center for ART treatment is about 32 years, and most patients were between 25 and 35 years old. Couples less than 25 years old seldom turn to ART treatment, unless there is a specific diagnosis reason of infertility. Advanced age females ( $>38$ year) were excluded in the study as well, therefore the absolute number of patients ranging from 25 to 35 was proportionally high, resulting in a potential sources of bias. The miscarriage rate was $14.2 \%$ in those younger than 25 years and $28.5 \%$ in those older than 3 years. These results demonstrate that the best reproductive age was between 25 to 35 years old.

In our study, about one third of the couples were had two-sided infections and two thirds had one-sided infections; however, the clinical outcomes regarding the success of ART therapy are comparable in these two subgroups, suggesting that $C$. trachomatis infection had no adverse effect, regardless of whether the infection was present in one or both partners.

\section{Conclusion}

Chlamydia trachomatis is common infection in subfertile people and it is critical to test for this organism before starting the ART process. The age range of infected individuals ranged between 25 to 35 years, and about one third of the couples had two sided infections that were transmitted sexually. For C. trachomatis infected patients, after a complete cure, there were no adverse effects on the clinical outcome, regardless of their gender, age group and number of copies.

\section{Abbreviations}

ART: assisted reproduction technique; C. trachomatis: Chlamydia trachomatis: PID: pelvic inflammatory disease; SAGE: serum protein substitute;

STD: sexually transmitted disease

\section{Acknowledgements}

We are very grateful to all participants in this study, and thank all the clinical staff. We also thank all the members of the Zhang and Zeng group for interesting discussions, helpful suggestions, and technical support.

\section{Funding}

This study was supported by the Science and Technology Program of Shenzhen (JCYJ20130401092000370 and JCYJ20120829150019349) and Medical Scientific Research Foundation of Guangdong Province (B2012331).

\section{Availability of data and materials}

Yes.

\section{Authors' contributions}

YOZ and YZ: study design and manuscript revise. YCZ: study design and manuscript write. THW and CMC: date collection. BY: data analysis. All authors read and approved the final manuscript.

\section{Competing interests}

The authors declare that they have no competing interests.

\section{Consent for publication}

The committee of ethical reviews the manuscript and consent for publication.

\section{Ethics approval and consent to participate}

The research project is met our ethic criterion and have been approved to perform strictly following the protocols, and informed consent was obtained from all couples prior to the study.

\section{Author details}

${ }^{1}$ School of Life Sciences, Tsinghua University, Beijing 100000, China. ${ }^{2}$ Key Lab in Healthy Science and Technology, Division of Life Science, Graduate School at Shenzhen, Tsinghua University, Shenzhen 518000, China. ${ }^{3}$ Central South University, the State Key Laboratory of Medical Genetics of China, Changsha 410000, China. ${ }^{4}$ Shenzhen Key Laboratory for Reproductive Immunology of Preimplantation, Shenzhen Zhongshan Institute for Reproduction and Genetics, Fertility Center, Shenzhen Zhongshan Urology Hospital, Shenzhen 518000, China.

Received: 7 July 2016 Accepted: 27 December 2016

Published online: 13 January 2017

\section{References}

1. Al-Fouzan A, Al-Mutairi N. Overview of incidence of sexually transmitted diseases in Kuwait. Clin Dermatol. 2004;22:509-12.

2. Wilson JS, Honey E, Templeton A. A systematic review of the prevalence of Chlamydia trachomatis among European women. Hum Reprod Update. 2002;8(4):385-94.

3. Sripada S, Amezaga MR, Hamilton M, McKenzie H, Templeton A, Bhattacharya S. Absence of chlamydial deoxyribonucleic acid from testicular and epididymal samples from men with obstructive azoospermia. Fertil Steril. 2010;93:833-6.

4. Peipert JF. Clinical practice. Genital chlamydial infections. N Engl J Med. 2003;349:2424-30.

5. de Vries HJ, Smelov V, Ouburg S, Pleijster J, Geskus RB, Speksnijder AG Fennema JS, Morre SA. Anal lymphogranuloma venereum infection screening with IgA anti-Chlamydia trachomatis-specific major outer membrane protein serology. Sex Transm Dis. 2010;37:789-95.

6. Jalal H, Stephen H, Al-Suwaine A, Sonnex C, Carne C. The superiority of polymerase chain reaction over an amplified enzyme immunoassay for the detection of genital chlamydial infections. Sex Transm Infect. 2006;82:37-40.

7. Stevens MP, Twin J, Fairley CK, Donovan B, Tan SE, Yu J, Garland SM, Tabrizi $\mathrm{SN}$. Development and evaluation of an ompA quantitative real-time PCR assay for Chlamydia trachomatis serovar determination. J Clin Microbiol. 2010;48:2060-5.

8. Malhotra M, Sood S, Mukherjee A, Muralidhar S, Bala M. Genital Chlamydia trachomatis: an update. Indian J Med Res. 2013;138:303-16. 
9. Haggerty CL, Gottlieb SL, Taylor BD, Low N, Xu F, Ness RB. Risk of sequelae after Chlamydia trachomatis genital infection in women. J Infect Dis. 2010;201 Suppl 2:S134-155.

10. Rusz A, Pilatz A, Wagenlehner F, Linn T, Diemer T, Schuppe HC, Lohmeyer J, Hossain H, Weidner W. Influence of urogenital infections and inflammation on semen quality and male fertility. World J Urol. 2012;30:23-30.

11. Trojian TH, Lishnak TS, Heiman D. Epididymitis and orchitis: an overview. Am Fam Physician. 2009;79:583-7.

12. Gallegos G, Ramos B, Santiso R, Goyanes V, Gosalvez J, Fernandez JL. Sperm DNA fragmentation in infertile men with genitourinary infection by Chlamydia trachomatis and Mycoplasma. Fertil Steril. 2008;90:328-34.

13. Eley A, Pacey AA, Galdiero M, Galdiero M, Galdiero F. Can Chlamydia trachomatis directly damage your sperm? Lancet Infect Dis. 2005;5:53-7.

14. Dixon RE, Hwang SJ, Hennig GW, Ramsey KH, Schripsema JH, Sanders KM, Ward SM. Chlamydia infection causes loss of pacemaker cells and inhibits oocyte transport in the mouse oviduct. Biol Reprod. 2009;80:665-73.

15. Scott $L$, Alvero R, Leondires M, Miller B. The morphology of human pronuclear embryos is positively related to blastocyst development and implantation. Hum Reprod. 2000;15:2394-403.

16. Fernandes LB, Arruda JT, Approbato MS, Garcia-Zapata MT. [Chlamydia trachomatis and Neisseria gonorrhoeae infection: factors associated with infertility in women treated at a human reproduction public service]. Rev Bras Ginecol Obstet. 2014;36:353-8.

17. Al-Mously N, Cross NA, Eley A, Pacey AA. Real-time polymerase chain reaction shows that density centrifugation does not always remove Chlamydia trachomatis from human semen. Fertil Steril. 2009:92:1606-15.

18. Dietrich W, Rath M, Stanek G, Apfalter P, Huber JC, Tempfer C. Multiple site sampling does not increase the sensitivity of Chlamydia trachomatis detection in infertility patients. Fertil Steril. 2010;93:68-71.

19. Yeow TC, Wong WF, Sabet NS, Sulaiman S, Shahhosseini F, Tan GM, Movahed E, Looi CY, Shankar EM, Gupta R, et al. Prevalence of plasmid-bearing and plasmid-free Chlamydia trachomatis infection among women who visited obstetrics and gynecology clinics in Malaysia. BMC Microbiol. 2016;16:45.

20. Parish WL, Laumann EO, Cohen MS, Pan S, Zheng H, Hoffman I, Wang T, $\mathrm{Ng} \mathrm{KH}$. Population-based study of chlamydial infection in China: a hidden epidemic. JAMA. 2003;289:1265-73.

21. Ramos BR, Polettini J, Marcolino LD, Vieira EP, Marques MA, Tristao AR, Nunes HR, Rudge MV, Silva MG. Prevalence and risk factors of Chlamydia trachomatis cervicitis in pregnant women at the genital tract infection in obstetrics unit care at Botucatu Medical School, Sao Paulo State UniversityUNESP, Brazil. J Low Genit Tract Dis. 2011;15:20-4.

22. Chen XS, Peeling RW, Yin YP, Mabey DC. The epidemic of sexually transmitted infections in China: implications for control and future perspectives. BMC Med. 2011:9:111.

23. Dielissen PW, Teunissen DA, Lagro-Janssen AL. Chlamydia prevalence in the general population: is there a sex difference? a systematic review. BMC Infect Dis. 2013;13:534.

\section{Submit your next manuscript to BioMed Central and we will help you at every step:}

- We accept pre-submission inquiries

- Our selector tool helps you to find the most relevant journal

- We provide round the clock customer support

- Convenient online submission

- Thorough peer review

- Inclusion in PubMed and all major indexing services

- Maximum visibility for your research

Submit your manuscript at www.biomedcentral.com/submit

) Biomed Central 